\title{
ON THE UPPER BOUND OF THE NUMBER OF REAL ROOTS OF A RANDOM ALGEBRAIC EQUATION WITH INFINITE VARIANCE. II
}

\author{
G. SAMAL AND M. N. MISHRA
}

\begin{abstract}
Let $N_{n}$ be the number of real roots of $\sum_{v=0}^{n} a_{v} \xi_{v} x^{v}=0$ where $\xi_{v}$ 's are independent random variables identically distributed with a common characteristic function $\exp \left(-C|t|^{\alpha}\right) ; C$ is a positive constant, $a_{0}, a_{1}, \cdots, a_{n}$ are nonzero real numbers such that $k_{n}=\max _{0} \leqq \nu \leqq n\left|a_{v}\right|=O\left(n^{\beta} / \log n\right)$. Then

(i) $\operatorname{Pr}\left\{\operatorname{Sup}_{n>n_{0}} N_{n} /(\log n)^{2}>\mu\right\}<\mu^{\prime} \mid n_{0}^{3 \alpha-2-\beta}, 1 \leqq \alpha \leqq 2,0<\beta<1$;

(ii) $\operatorname{Pr}\left\{N_{n} /(\log n)^{2}>\mu\right\}<\mu^{\prime} \mid n, \alpha \geqq 1$;

(iii) $\operatorname{Pr}\left\{N_{n} /(\log n)^{2}>\mu\right\}<\mu^{\prime} / n^{3 \alpha-1}-\beta, 1 \leqq \alpha \leqq 2$.
\end{abstract}

1. Introduction. In our paper [2] we have considered the upper bound of the number of real roots of the algebraic equation

$$
f(x)=\sum_{v=0}^{n} \xi_{v} x^{v}=0
$$

whose coefficients $\xi_{v}$ 's are identically distributed independent random variables with a common characteristic function $\exp \left(-C|t|^{\alpha}\right)$ where $C$ is a positive constant and $\alpha \geqq 1$. In fact we have proved that

$$
\operatorname{Pr}\left\{\operatorname{Sup}_{n>n 0} N_{n} /(\log n)^{2}>\mu\right\}<\mu^{\prime} / n_{0}^{3 \alpha-2-\beta}, \quad 0<\beta<1,1 \leqq \alpha \leqq 2
$$

$$
\begin{array}{ll}
\operatorname{Pr}\left\{N_{n} /(\log n)^{2}>\mu\right\}<\mu^{\prime} / n, & \alpha \geqq 1 ; \\
\operatorname{Pr}\left\{N_{n} /(\log n)^{2}>\mu\right\}<\mu^{\prime} / n^{3 \alpha-1-\beta}, & 1 \leqq \alpha \leqq 2,
\end{array}
$$

where $N_{n}$ is the number of real roots of $f(x)=0$.

In the present work we like to point out that there is no need of taking the coefficients as identically distributed and that our result could be put in more general form.

In the place of equation (1) we shall consider the equation

$$
f(x)=\sum_{v=0}^{n} a_{v} \xi_{v} x^{v}=0
$$

Received by the editors December 25, 1972 and, in revised form, September 6, 1973. AMS (MOS) subject classifications (1970). Primary 60-XX.

Key words and phrases. Random variables, infinite variance, real roots, random algebraic equations.

(c) American Mathematical Society 1974 
where $a_{0}, a_{1}, \cdots, a_{n}$ are nonzero real numbers with some restrictions. The coefficients $a_{v} \xi_{v}$ 's are not identically distributed although $\xi_{v}$ 's have the same distribution.

Equations of the type (2) have been considered by Dunnage [1], but his variance is finite whereas our variance is infinite for $1<\alpha<2$.

In our paper [3] we have considered the corresponding result for the lower bound.

2. THEOREM 1. Let $f(x)=\sum_{v=0}^{n} a_{v} \xi_{v} x^{v}$ be a polynomial where $\xi_{v}$ 's are independent random variables identically distributed with a common characteristic function $\exp \left(-C|t|^{\alpha}\right), C$ is a positive constant and $1 \leqq \alpha \leqq 2 . a_{0}, a_{1}$, $a_{2}, \cdots, a_{n}$ are nonzero real numbers such that $k_{n}^{\alpha}=O\left(n^{\beta} / \log n\right), 0<\beta<1$ where $k_{n}=\max _{0 \leqq v \leqq n}\left|a_{v}\right|, t_{n}=\min _{0 \leqq v \leqq n}\left|a_{v}\right|$. Then there exists an integer $n_{0}$ such that for each $n>n_{0}$, the number of real roots of the equation $f(x)=0$ is at most $\mu(\log n)^{2}$ except for a set of measure at most $\mu^{\prime} / n_{0}^{3 \alpha-2-\beta}$.

Proof. We shall indicate only the modifications necessary in the proof of Theorem 1 of our paper cited above. The steps not mentioned here remain unchanged.

In $\S 5$, we shall have

Hence

$$
\operatorname{Pr}\left\{\left|a_{v} \xi_{v}\right| \geqq(n+1)^{3}\right\} \leqq \mu\left|a_{v}\right|^{\alpha} /(n+1)^{3 \alpha} .
$$

$$
\begin{aligned}
\operatorname{Pr}\left\{\left|a_{v} \xi_{v}\right|<(n+1)^{3}, 0 \leqq v \leqq n\right\} & >1-\left(\mu \sum_{0}^{n}\left|a_{v}\right|^{\alpha}\right) /(n+1)^{3 \alpha} \\
& >1-\mu K_{n}^{\alpha} /(n+1)^{3 \alpha-1} .
\end{aligned}
$$

So outside a set of measure at most $\mu K_{n}^{\alpha} /(n+1)^{3 \alpha-1}$,

$$
\max _{|z| \leqq 1+2 / n}|f(z)| \leqq C^{2}(n+1)^{4} .
$$

Now, $\operatorname{Pr}\left\{\left|\xi_{v}\right|<1 / n^{5}\right\} \leqq \mu / C^{1 / \alpha} n^{5}$. Since the characteristic function of $f\left(x_{m}\right)$ is $\exp \left(-C|t|^{\alpha} \sum_{v=0}^{n}\left|a_{v}\right|^{\alpha} x_{m}^{\alpha \nu}\right)$ we have

$$
\operatorname{Pr}\left\{\left|f\left(x_{m}\right)\right|<1 / n^{5}\right\} \leqq \frac{\mu}{n^{5}}\left(\sum_{\nu=0}^{n}\left|a_{\nu}\right|^{\alpha} x_{m}^{\alpha \nu}\right)^{-1 / \alpha}<\frac{\mu}{t_{n} n^{5}}\left(\sum x_{m}^{\alpha \nu}\right)^{-1 / \alpha}
$$

for $m=1,2, \cdots, k, p \log n$ and

$$
\operatorname{Pr}\left\{\left|f\left(x_{0}\right)\right|<1 / n^{5}\right\} \leqq \frac{\mu}{n^{5}}\left(\sum_{v=0}^{n}\left|a_{v}\right|^{\alpha}\right)^{-1 / \alpha}<\mu /\left(n^{5+1 / \alpha} \cdot t_{n}\right)
$$

for $m=0$.

So the number of zeros of $f(z)$ in all the circles $C_{0}, C_{1}, \cdots, C_{k}, C_{p \log n}$ is at most $\mu(\log n)^{2}$. 
The measure of the exceptional set is at most

$$
\begin{aligned}
\frac{\mu k_{n}^{\alpha} \log n}{(n+1)^{3 \alpha-1}}+\frac{\mu}{t_{n} \cdot n^{5}} & \sum_{m=1}^{p \log n}\left(\sum_{v=0}^{n} x_{m}^{\alpha \nu}\right)^{-1 / \alpha}+\frac{\mu}{n^{5+1 / \alpha} \cdot t_{n}} \\
& <\frac{\mu k_{n}^{\alpha} \log n}{(n+1)^{3 \alpha-1}}+\frac{\mu \log n}{t_{n} \cdot n^{5+1 / \alpha}}+\frac{\mu}{t_{n} \cdot n^{5}}+\frac{\mu}{n^{5+1 / \alpha} \cdot t_{n}} \\
& <\frac{\mu k_{n}^{\alpha} \log n}{(n+1)^{3 \alpha-1}}+\frac{\mu}{t_{n} \cdot n^{5}}<\frac{\mu}{n^{3 \alpha-1-\beta}},
\end{aligned}
$$

since $k_{n}^{\alpha}=O\left(n^{\beta} / \log n\right)$ and without loss of generality we could suppose $t_{n} \geqq 1$. The measure of the exceptional set corresponding to the segment $\left[0, \frac{1}{2}\right]$ is at most

$$
\mu k_{n}^{\alpha} /(n+1)^{3 \alpha-1}+\mu / n^{5}<\mu / n^{3 \alpha-1-\beta} .
$$

There is no change in the remaining part of the proof.

3. THEOREM 2. Let $N_{n}$ be the number of real roots of the equation $f(x)=0$ satisfying the conditions of Theorem 1 , where $\alpha \geqq 1$. Then $N_{n}<\mu(\log n)^{2}$ outside a set of measure at most $\mu^{\prime} / n$.

Proof. In $\S 6$ of our paper,

and

$$
\operatorname{Pr}\left\{\left|a_{v} \xi_{v}\right| \geqq(n+1)^{3}\right\} \leqq \mu\left|a_{v}\right|^{\alpha} /(n+1)^{3 \alpha}
$$

$$
\operatorname{Pr}\left\{\left|\xi_{v}\right|<1 / n\right\} \leqq \mu / C^{1 / \alpha} n \text {. }
$$

So the measure of the exceptional set is at most

$$
\begin{aligned}
\frac{\mu k_{n}^{\alpha} \log n}{(n+1)^{3 \alpha-1}}+\frac{\mu}{t_{n}} \frac{\log n}{n^{1+1 / \alpha}}+\frac{\mu}{t_{n} \cdot n} & +\frac{\mu}{n^{1+1 / \alpha} \cdot t_{n}} \\
& <\mu k_{n}^{\alpha} \log n /(n+1)^{3 \alpha-1}+\mu /\left(t_{n} \cdot n\right) \\
& <\mu / n^{3 \alpha-1-\beta}+\mu / n<\mu / n
\end{aligned}
$$

since $t_{n} \geqq 1$ and $k_{n}^{\alpha}=O\left(n^{\beta} / \log n\right)$.

\section{REFERENCES}

1. J. E. A. Dunnage, The number of real zeros of a class of random algebraic polynomials. II, Quart. J. Math. Oxford Ser. (2) 21 (1970), 309-319. MR 43 \#1239.

2. G. Samal and M. N. Mishra, On the upper bound of the number of real roots of $a$ random algebraic equation with infinite variance, J. London Math. Soc. 6 (1973), 598604.

3. - On the lower bound of the number of real roots of a random algebraic equation with infinite variance. III, Proc. Amer. Math. Soc. 39 (1973), 184-189.

Department of Mathematics, Ravenshaw College, Cuttack 3, Orissa, India (Current address of G. Samal)

Current address (M. N. Mishra): Bureau of Statistics, Bhubaneswar, Orissa, India 